\title{
Election Result Prediction System using Hidden Markov Model [HMM]
}

\author{
Mohd. Manjur Alam \\ Asst. Professor \\ Dept. of CSE \\ BGC Trust University Bangladesh
}

\author{
Md. MezbahUddin \\ Student \\ Dept. of CSE \\ BGC Trust University Bangladesh
}

\author{
Shamsun Nahar Shoma \\ Lecturer \\ Dept. of CSE \\ BGC Trust University Bangladesh
}

\begin{abstract}
Election is an important part of political and social science. It can be defined in the field of Game as the winning chance of a team and TV reality show where candidates are the participants and decide if the participants will stay or not based on public votes. The election result can be predicted before the actual outcome using a prediction method. There are many methods, theory, and research to predict election result. Election prediction is very significant for the candidates and the society. It is normally based on some factors such as numbers of years in active politics, Popularity, Vote Bank, Development performance, Currently in Govt., View of voters towards party, Major Issue, Party/Independent and Internal War. In this paper a most famous model named Hidden Markov Model has been used to predict the results using these parameters.
\end{abstract}

\section{Keywords}

Hidden Markov Model (HMM), Election Commission of Bangladesh [ECB].

\section{INTRODUCTION}

In the Earth, Election is very much liking word. It is a method to select a qualified one from a crowd of candidates. But in different sector election process may be different. There are different types of election such as parliamentary election, legislative election, local election, presidential election and election in small group. It can be divided into two categories. The election in a small organization or group or within an institution can be classified into a group, and Country election such as Parliamentary election, Presidential election, election for mayor and councilor in municipal area, Union Perished election etc. can be classified into another group. In Bangladesh there are 8 divisions, 65 districts and each district has an individual number of sub-district and Thaana. Every sector has election system to select a leader such as Mayor, Chairman, and Councilor etc. Different organizations use different electoral methods to compute the election. ECB evaluates the election in those field related to the government, District, Thana. Election prediction is also very important as like as election. But it is not so easy to predict election result and has a lot of challenges to predict election result. Hidden Markov Model [HMM] is used to evaluate the prediction. Above nine parameters will be used to predict the chances of winning and losing chances of a candidate.

\section{FACTOR DESCRIPTION}

There are some factors which may influence the mode of an election system. These are shown in table 1 with descriptions.
Table 1: Factor Description

\begin{tabular}{|c|c|}
\hline Factor Name & Factor Description \\
\hline $\begin{array}{c}\text { No of years in } \\
\text { active politics }\end{array}$ & Political experience under any registered \\
party.
\end{tabular}

\section{HIDDEN MARKOV MODEL FOR PREDICTION IN ELECTION SYSTEM}

A Hidden Markov Model (HMM) is an automated system with output where both the state transition and the output are defined in a probabilistic manner. The state Transition arises according to a simple Markov model but it is assumed that we don't know on which state we are standing now and that we can observe an output symbol at each state. We could estimate the transition sequences through observing output sequence. In this thesis, The most flexible and successful approach, the Hidden Markov Model (HMM) method as the training/recognition algorithm [1] for prediction in an election system has been used. The objectives of HMM parameter estimation is to maximize the likelihood of the data under the 
given parameter setting. The three basic parameters in HMM are given below:

1. $\pi$ - The initial state distribution.

2. a- The state-transition probability matrix.

3. B - Observation probability distribution.

\subsection{Estimating The Parameters, $\lambda=$} $(A, B, \pi)$

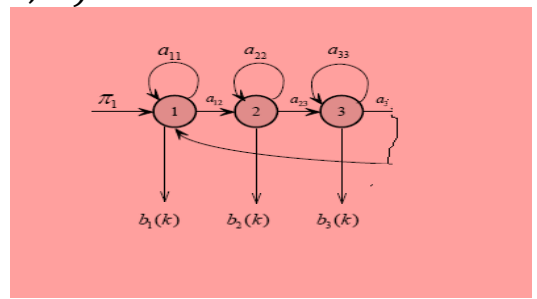

Fig.1 : HMM for each Candidate.

The model parameters can be estimated as follows:

$$
\begin{aligned}
& \pi_{i} \\
& =\gamma_{1}(i)
\end{aligned}
$$

$=$ expected number of times in state $\mathrm{i}$

$$
=\frac{\sum_{t=1}^{T-1} \xi_{t}(i, j)}{\sum_{t=1}^{T-1} \gamma_{t}(i)}
$$

$=\frac{\text { expectednumberoftransitionfromstateitostate } j}{\text { expected number of transition from state } i}$

\section{$\mathbf{b}_{\mathbf{i}}(\mathbf{k})$ Measurement}

Probability from each state to observation is measured from winning probability of each factor in the election. It is actually evaluated from the influencing of the factor in the election system.

\subsection{Scores}

For each candidate, probability score is computed [first]. The candidate whose model produces the highest probability score is selected as the winner. The total probability is given by:

$\mathrm{P}(\mathrm{X} 1 \mathrm{Q})=\prod_{i=1, j=1}^{n} \mathrm{a} \quad{ }_{\mathrm{ij}} \mathrm{b}_{\mathrm{i}}(\mathrm{k})$

\section{EXPERIMENTAL RESULT}

Bangladesh is a democratic country. The people of this country strongly believe the democracy. So the election system of every sector in our country is very neutral and fair. Many organizations conduct many surveys for prediction in the election system. Here some data before the election are gathered from the observations of B. M. Char Union Council election, 2012. The union is situated at Cox'sBazar in Bangladesh. The survey has been accomplished by SSSD. The data about the Chairman candidates are collected for the prediction. There are five candidates for the post. The candidates are Mr.Shahedul karim, Mr.MuslehUddin, Mr.Nuhan Jakir, Mr. MesbahUddin and Mr. Nurul hossain. Factors are described in table 1 for the prediction. The organization estimated the influencing factors for the each chairman candidate. These are mentioned in table 2 . Popularities and vote bank are evaluated among 100 people here.

\begin{tabular}{|c|c|c|c|c|c|}
\hline $\begin{array}{l}\text { Factor } \\
\text { Name }\end{array}$ & $\begin{array}{c}\text { Shahedul } \\
\text { karim }\end{array}$ & $\begin{array}{l}\text { Musleh } \\
\text { Uddin }\end{array}$ & $\begin{array}{c}\text { Nuhan } \\
\text { Jakir }\end{array}$ & $\begin{array}{c}\text { MesbahU } \\
\text { ddin }\end{array}$ & $\begin{array}{c}\text { Nurul } \\
\text { Hossain }\end{array}$ \\
\hline $\begin{array}{c}\text { No of } \\
\text { years in } \\
\text { active } \\
\text { politics(wit } \\
\text { h linguistic } \\
\text { Values ) }\end{array}$ & 10 & .4 & .5 & .6 & $\begin{array}{l}2 \\
.1\end{array}$ \\
\hline Popularity & $23 \%$ & $25 \%$ & $12 \%$ & $15 \%$ & $15 \%$ \\
\hline Vote Bank & $\begin{array}{c}12 \% \\
\text { (most } \\
\text { Hindus) }\end{array}$ & $\begin{array}{c}13 \% \\
\text { (Most } \\
\text { Muslims) }\end{array}$ & $\begin{array}{c}10 \% \\
\text { (Hindu, } \\
\text { Muslims } \\
\text { ) }\end{array}$ & $\begin{array}{c}30 \% \\
\text { (Most } \\
\text { Hindus) }\end{array}$ & $\begin{array}{c}34 \% \\
\text { (Most } \\
\text { Muslim } \\
\text { s) }\end{array}$ \\
\hline $\begin{array}{c}\text { Developm } \\
\text { ent } \\
\text { performan } \\
\text { ce } \\
\text { (with } \\
\text { linguistic } \\
\text { Values ) }\end{array}$ & $\begin{array}{c}\text { Low, } \\
.1\end{array}$ & $\begin{array}{c}\text { Low } \\
.1\end{array}$ & $\begin{array}{c}\text { Medium } \\
.2\end{array}$ & $\begin{array}{c}\text { High } \\
.3\end{array}$ & $\begin{array}{c}\text { High } \\
.3\end{array}$ \\
\hline $\begin{array}{l}\text { View of } \\
\text { voters } \\
\text { towards } \\
\text { party } \\
\text { (with } \\
\text { linguistic } \\
\text { Values) }\end{array}$ & $\begin{array}{c}\text { Average } \\
.4\end{array}$ & $\begin{array}{c}\text { Average } \\
.4\end{array}$ & $\begin{array}{c}\text { Average } \\
.4\end{array}$ & $\begin{array}{c}\text { Average } \\
.4\end{array}$ & $\begin{array}{c}\text { Averag } \\
\text { e } \\
.4\end{array}$ \\
\hline $\begin{array}{l}\text { Currently } \\
\text { in Govt. } \\
\text { (with } \\
\text { linguistic } \\
\text { Values) }\end{array}$ & $\begin{array}{c}\text { No } \\
.1\end{array}$ & $\begin{array}{l}\text { No } \\
.1\end{array}$ & $\begin{array}{c}\text { Yes } \\
.2\end{array}$ & $\begin{array}{c}\text { Yes } \\
.2\end{array}$ & $\begin{array}{c}\text { Yes } \\
.2\end{array}$ \\
\hline $\begin{array}{c}\text { Major } \\
\text { Issue } \\
\text { (with } \\
\text { linguistic } \\
\text { Values) }\end{array}$ & $\begin{array}{c}\text { Null } \\
.1\end{array}$ & $\begin{array}{c}\text { Null } \\
.1\end{array}$ & $\begin{array}{c}\text { Null } \\
.1\end{array}$ & $\begin{array}{c}\text { Null } \\
.1\end{array}$ & $\begin{array}{c}\text { Null } \\
.1\end{array}$ \\
\hline $\begin{array}{c}\text { Party/Inde } \\
\text { pendent } \\
\text { (with } \\
\text { linguistic } \\
\text { Values) }\end{array}$ & $\begin{array}{c}\text { Yes } \\
.3\end{array}$ & $\begin{array}{c}\text { No } \\
.1\end{array}$ & $\begin{array}{c}\text { No } \\
.1\end{array}$ & $\begin{array}{c}\text { Yes } \\
.3\end{array}$ & $\begin{array}{c}\text { No } \\
.3\end{array}$ \\
\hline $\begin{array}{l}\text { Internal } \\
\text { War } \\
\text { (with } \\
\text { linguistic } \\
\text { Values) }\end{array}$ & $\begin{array}{c}\text { Yes } \\
.4\end{array}$ & $\begin{array}{l}\text { No } \\
.1\end{array}$ & $\begin{array}{c}\text { No } \\
.1\end{array}$ & $\begin{array}{c}\text { Average } \\
.3\end{array}$ & $\begin{array}{c}\text { No } \\
.3\end{array}$ \\
\hline
\end{tabular}

\subsection{Hi Estimation}

$\pi_{1}=.1, \pi_{2}==.1, \pi_{3}=.1=, \pi_{4}==.1, \pi_{5}=.1$,

Table 2: probabilities of factors

\section{2 $\mathrm{a}_{\mathrm{ij}}$ Estimation}

Each HMM has nine states for nine factors and nine observations

Hence, $a_{11}=a_{12}=a_{22}=a_{23}=a_{33}=a_{34}=a_{44}=a_{45}=a_{55}$ 
$=a_{56}=a_{66}=a_{67}=a_{77}=a_{78}=a_{88}=a_{89}=a_{99}=a_{91}=1 / 2=.5$;

\section{$4.3 b_{i}(k)$ Measurement}

From the survey mentioned in section $4, \boldsymbol{b}_{\boldsymbol{i}}(\boldsymbol{k})$ can be easily evaluated. It is done by measuring influencing probability of each factor which is shown in table 2. For each candidate, these are evaluated below:

Mr. Shahedul karim:

$\mathrm{b}_{1}(\mathrm{k})=.9, \mathrm{~b}_{2}(\mathrm{k})=.23, \mathrm{~b}_{3}(\mathrm{k})=.12$,

$\mathrm{b}_{4}(\mathrm{k})=.1, \mathrm{~b}_{5}(\mathrm{k})=.4, \mathrm{~b}_{6}(\mathrm{k})=.1$,

$\mathrm{b}_{7}(\mathrm{k})=.1, \mathrm{~b}_{8}(\mathrm{k})=.3, \mathrm{~b}_{9}(\mathrm{k})=.4$,

Mr. MuslehUddin:

$\mathrm{b}_{1}(\mathrm{k})=.4, \mathrm{~b}_{2}(\mathrm{k})=.25, \mathrm{~b}_{3}(\mathrm{k})=.13$,

$\mathrm{b}_{4}(\mathrm{k})=.1, \mathrm{~b}_{5}(\mathrm{k})=.4, \mathrm{~b}_{6}(\mathrm{k})=.1$,

$\mathrm{b}_{7}(\mathrm{k})=.1, \mathrm{~b}_{8}(\mathrm{k})=.1, \mathrm{~b}_{9}(\mathrm{k})=.1$,

Mr. Nuhan Jakir:

$\mathrm{b}_{1}(\mathrm{k})=.5, \mathrm{~b}_{2}(\mathrm{k})=.12, \mathrm{~b}_{3}(\mathrm{k})=.1$,

$\mathrm{b}_{4}(\mathrm{k})=.2, \mathrm{~b}_{5}(\mathrm{k})=.4, \mathrm{~b}_{6}(\mathrm{k})=.2$,

$\mathrm{b}_{7}(\mathrm{k})=.1, \mathrm{~b}_{8}(\mathrm{k})=.1, \mathrm{~b}_{9}(\mathrm{k})=.1$,

Mr. Mesbah Uddin:

$\mathrm{b}_{1}(\mathrm{k})=.6, \mathrm{~b}_{2}(\mathrm{k})=.15, \mathrm{~b}_{3}(\mathrm{k})=. .30$,

$\mathrm{b}_{4}(\mathrm{k})=.3, \mathrm{~b}_{5}(\mathrm{k})=.4, \mathrm{~b}_{6}(\mathrm{k})=.2$,

$\mathrm{b}_{7}(\mathrm{k})=.1, \mathrm{~b}_{8}(\mathrm{k})=.3, \mathrm{~b}_{9}(\mathrm{k})=.3$,

Mr. Nurul hossain:

$\mathrm{b}_{1}(\mathrm{k})=.1, \mathrm{~b}_{2}(\mathrm{k})=.15, \mathrm{~b}_{3}(\mathrm{k})=.34$,

$\mathrm{b}_{4}(\mathrm{k})=.3, \mathrm{~b}_{5}(\mathrm{k})=.4, \mathrm{~b}_{6}(\mathrm{k})=.2$,

$\mathrm{b}_{7}(\mathrm{k})=.1, \mathrm{~b}_{8}(\mathrm{k})=.3, \mathrm{~b}_{9}(\mathrm{k})=.3$,

\subsection{Scores Estimation}

The individual candidate scores are shown on the following tables. The scores are computed by using the formula given in Eq.(3)

\subsubsection{Mr. Shahedul karim}

Table 3: Mr. Shahedul karim

\begin{tabular}{|l|l|l|l|}
\hline $\begin{array}{l}\text { State } \\
\text { seq } \\
\text { uen } \\
\text { ce } \\
(\mathbf{Q})\end{array}$ & $\begin{array}{c}\text { Observation } \\
\text { sequen } \\
\text { ce(x) }\end{array}$ & $\begin{array}{l}\text { State } \\
\text { transition } \\
\text { probabilit } \\
\mathbf{y}, \mathrm{a}_{\mathrm{ij}}\end{array}$ & $\begin{array}{l}\text { Observation } \\
\text { probabil } \\
\text { ities, } \\
\mathrm{b}_{\mathrm{i}}(\mathrm{k})\end{array}$ \\
\hline Q1 & $\mathbf{1}$ & .5 & .9 \\
\hline Q2 & 2 & .5 & .23 \\
\hline Q3 & $\mathbf{3}$ & .5 & .12 \\
\hline Q4 & 4 & .5 & .1 \\
\hline Q5 & $\mathbf{5}$ & .5 & .4 \\
\hline Q6 & 6 & .5 & .1 \\
\hline Q7 & 7 & .5 & .1 \\
\hline Q8 & $\mathbf{8}$ & .5 & .3 \\
\hline Q9 & 9 & .5 & .4 \\
\hline
\end{tabular}

Total probability for Mr. Shahedul karim, $\mathrm{P}(\mathrm{X} 1 \mathrm{Q})=$ 0.0000000023

\subsubsection{Mr. Musleh Uddin}

Table 4:Mr. Musleh Uddin

\begin{tabular}{|c|c|c|c|}
\hline $\begin{array}{c}\text { State } \\
\text { seq } \\
\text { uen } \\
\text { ce } \\
(\text { Q) }\end{array}$ & $\begin{array}{c}\text { Observation } \\
\text { sequen } \\
\text { ce(x) }\end{array}$ & $\begin{array}{c}\text { State } \\
\text { transi } \\
\text { tion } \\
\text { proba } \\
\text { bilitya } \\
\text { ij }\end{array}$ & $\begin{array}{c}\text { Observation } \\
\text { probabilit } \\
\text { ies, } \\
\mathrm{b}_{\mathrm{i}}(\mathrm{k})\end{array}$ \\
\hline Q1 & 1 & .5 & .4 \\
\hline Q2 & 2 & .5 & .25 \\
\hline Q3 & 3 & .5 & .13 \\
\hline Q4 & 4 & .5 & .1 \\
\hline Q5 & 5 & .5 & .4 \\
\hline Q6 & 6 & .5 & .1 \\
\hline Q7 & 7 & .5 & .1 \\
\hline Q8 & 8 & .5 & .1 \\
\hline Q9 & 9 & .5 & .1 \\
\hline
\end{tabular}

Total probability for Mr. Musleh Uddin, $\mathrm{P}(\mathrm{X} 1 \mathrm{Q})=$ 0.0000000008

\subsubsection{Mr. Nuhan Jakir}

Table 5:Mr. Nuhan Jakir

\begin{tabular}{|l|l|l|l|}
\hline $\begin{array}{l}\text { State } \\
\text { seq } \\
\text { uen } \\
\begin{array}{c}\text { ce } \\
(\text { Q) }\end{array}\end{array}$ & $\begin{array}{l}\text { Observation } \\
\text { sequen } \\
\text { ce(x) }\end{array}$ & $\begin{array}{l}\text { State } \\
\text { transiti } \\
\text { on } \\
\text { probab } \\
\text { ility, } \mathrm{a}_{\mathrm{ij}}\end{array}$ & $\begin{array}{l}\text { Observation } \\
\text { probabil } \\
\text { ities, }\end{array}$ \\
\hline Q1 & 1 & .5 & .5 \\
\hline Q2 & 2 & .5 & .12 \\
\hline Q3 & 3 & .5 & .1 \\
\hline Q4 & 4 & .5 & .2 \\
\hline Q5 & 5 & .5 & .4 \\
\hline Q6 & 6 & .5 & .2 \\
\hline Q7 & 7 & .5 & .1 \\
\hline Q8 & 8 & .5 & .1 \\
\hline Q9 & 9 & .5 & .1 \\
\hline
\end{tabular}

Total probability for Mr. Nuhan Jakir,P(X1Q) = 0.0000000002

\subsubsection{Mr. Mesbah Uddin \\ Table 6:Mr. Mesbah Uddin}

\begin{tabular}{|c|c|c|c|}
\hline $\begin{array}{c}\text { State } \\
\text { sequence } \\
(\mathbf{Q})\end{array}$ & $\begin{array}{c}\text { Observation } \\
\text { sequence(x) }\end{array}$ & $\begin{array}{c}\text { State } \\
\text { transition } \\
\text { probability, } \\
\mathrm{a}_{\mathrm{ij}}\end{array}$ & $\begin{array}{c}\text { Observation } \\
\text { probabilities, } \\
\mathrm{b}_{\mathrm{i}}(\mathrm{k})\end{array}$ \\
\hline Q1 & 1 & .5 & .6 \\
\hline Q2 & 2 & .5 & .15 \\
\hline
\end{tabular}




\begin{tabular}{|c|c|c|c|}
\hline Q3 & 3 & .5 & .3 \\
\hline Q4 & 4 & .5 & .3 \\
\hline Q5 & 5 & .5 & .4 \\
\hline Q6 & 6 & .5 & .2 \\
\hline Q7 & 7 & .5 & .1 \\
\hline Q8 & 8 & .5 & .3 \\
\hline Q9 & 9 & .5 & .3 \\
\hline
\end{tabular}

Total probability for Mr. Mesbah Uddin, $\mathrm{P}(\mathrm{X} 1 \mathrm{Q})=$ 0.0000000114

\subsubsection{Mr. Nurul Hossain}

Table 7:Mr. Nurul Hossain

\begin{tabular}{|c|c|c|c|}
\hline $\begin{array}{c}\text { State } \\
\text { sequence } \\
(\mathbf{Q})\end{array}$ & $\begin{array}{c}\text { Observation } \\
\text { sequence(x) }\end{array}$ & $\begin{array}{c}\text { State } \\
\text { transition } \\
\text { probability, } \\
\mathrm{a}_{\mathrm{ij}}\end{array}$ & $\begin{array}{c}\text { Observation } \\
\text { probabilities } \\
, \\
\mathrm{b}_{\mathrm{i}}(\mathrm{k})\end{array}$ \\
\hline Q1 & $\mathbf{1}$ & .5 & .1 \\
\hline Q2 & 2 & .5 & .15 \\
\hline Q3 & $\mathbf{3}$ & .5 & .34 \\
\hline Q4 & 4 & .5 & .3 \\
\hline Q5 & 5 & .5 & .4 \\
\hline Q6 & 6 & .5 & .2 \\
\hline Q7 & 7 & .5 & .1 \\
\hline Q8 & $\mathbf{5}$ & .5 & .3 \\
\hline Q9 & 9 & .5 & .3 \\
\hline
\end{tabular}

Total probability for Mr. Nurul Hossain, $\mathrm{P}(\mathrm{X} 1 \mathrm{Q})=$ 0.0000000022

\section{RESULT}

The highest score is obtained from Mesbah Uddin, hence the winner will be Mesbah Uddin in this election.

\section{CONCLUSION AND FUTURE WORK}

This research work computes the winning probability of a candidate. Some factors / parameters are used for this proposed work. These factors are taken and discussed from experts in the area of political science. The purpose behind this research is to do prediction for a candidate without using only public opinion. For this purpose, concept of Hidden Markov Model [HMM] has used. Some factors related to

election have been chosen because these factors effects election. The future work of this research is that more number of parameters can be used to predict the result. Moreover these parameters can be changed as per the geographical area of constituency. Even the effect to time is also a responsible factor in election. New input parameters can be added or edited according to the time. Increment in sample size will also lead to give more accurate result.

\section{REFERENCES}

[1] Text Dependent Speaker Identification using Hidden Markchov Model and Mel Frequency Cepstrum Coefficient,Mohd. ManjurAlam, Md. Salah UddinChowdury, NiazUddin Mahmud, BGC Trust University Bangladesh, International Journal of Computer Applications (0975 - 8887) Volume 104 No.14, October 2014.

[2] Using voter-choice modeling to plan the final campaign in runoff elections, Wagner A. Kamakura, Rice University.

[3] Election Results Prediction System based on Fuzzy Logic, Harmanjit Singh Research Scholar, Singhania University,Rajasthan, INDIA Gurdev Singh, Ph.D Professor, Deptt of Information\& Technology, Gurukul Vidyapeeth Institute of Engineering \& Technology Banur, Chandigarh, Nitin Bhatia DAV College, Jalandhar Punjab, INDIA. International Journal of Computer Applications ((0975 - 8887) Volume 53- No.9, September 201214.

[4] Text-Independent Speaker Identification Using Hidden Markov Model SayedJaaferAbdallah, Izzeldin Mohamed Osman, Mohamed Elhafiz Mustafa, College of Computer Science and Information Technology Sudan University of Science and Techn, ogy, "World of Computer Science and Information Technology Journal (WCSIT) ISSN: 2221-0741 Vol. 2, No. 6, 203-208, 2012 Khartoum, Sudan.

[5] Predicting Election Popularity of a Person Using Crowd Sensing In Social Networks, Bangladesh. Mashroora Nadi,

[6] Syed Washfi Ahmad, S.M. Saquib Rahman, Brac University.

[7] Tumasjan, A., Sprenger, T., Sandner, P., \& Welpe, I. (n.d.). Predicting Elections with Twitter: What 140 Characters Reveal about Political Sentiment.

[8] McCarthy, Colm and Terence M. Ryan (1977) "Estimates of Voter Transition Probabilities from the British General Elections of 1974," Journal of the Royal Statistical Society. Series A, 140(1). 